\title{
Knowledge Management For Best Practices
}

\author{
Daniel E. O’Leary and Peter Selfridge
}

Perhaps one of the most celebrated and significant recent developments in information systems in business has been business process reengineering (BPR; e.g., Hammer 1990 and Davenport 1993). Businesses now have entire departments of reengineering with vice presidents of reengineering or their equivalent reported in about half of Fortune 500 companies. Staffs in those departments have been reported to be as large as 150 people. 1 Business process reengineering has ubstantial support for reengineering efforts has been incorporated into reengineering tools.2 Recently re-searchers have focused on using artificial intelligence (AI) in developing those tools.3 However, little research has been conducted on the extent to which those research developments have been captured in current reengineering tools. In addition, the research literature on the application of AI to reengineering is just being developed, and some aspects of reengineering are not addressed. For example, few knowledge-based systems focus on capturing the knowledge necessary to reengineer a specific process or even help decide whether a company is ready to launch a reengineering project.

Given this context, in this article we

- Define reengineering;

- Investigate current usage of AI in reengineering tools, based on a survey of tool developers;

- Review existing research literature on the use of AI to facilitate and support reengineering efforts; and

- Discuss a particular "best practices" knowledge-based system designed to facilitate and support reengineering of procurement systems. This system is based on the acquisition and categorization of relevant procurement knowledge and demonstrates that knowledge-based systems can be an important part of knowledge management.

As a result, this article will identify many of the important efforts designed to integrate AI with reengineering tools. The discussion will be of interest to those who have identified the need or desire to embed AI into reengineering tools. It will also help those who are interested in the benefits of embedding AI into reengineering tools. Finally, it provides a general introduction to reengineering business processes and the support of reengineering using computer-based tools.

DANiEL E. O’Leary (oleary@rcf.usc.edu) University of Southern California, 3660 Trousdale Parkway, Los Angeles, CA.

Peter Selfridge AT\&T Labs-Research, 600 Mountain Avenue, Murray Hill, NJ.

Permission to make digital or hard copies of all or part of this work for personal or classroom use is granted without fee provided that copies are not made or distributed for profit or commercial advantage and that copies bear this notice and the full citation on the first page. To copy otherwise, to republish, to post on servers or to redistribute to lists, requires prior specific permission and/or a fee.

(C) 2000 ACM 0002-0782/00/1100 \$5.00 


\section{What Is Reengineering?}

Business process reengineering, or simply, reengineering, is the use of modern technology to redesign business processes, often radically, in order to achieve dramatic improvements in performance (Hammer 1990, p. 104). Reengineering focuses on using information technology (IT) in new ways so that processes are redesigned instead of automated. Rather than simply replicating existing processes in different IT environments, reengineering attempts to exploit technologies to change processes.

As part of his analysis, Hammer (1990) listed seven principles of reengineering. These are one view of the core general concepts for reengineering a process.

- Organize around outcomes, not tasks. If a process is organized as a set of tasks, the primary concern will be the set of task outputs rather than the overall outcome. Task-based organization tends to preserve the old way of doing things. For example, reengineering means that rather than having multiple people each do a small part of the process, one person might do all the steps in a particular process.

- Have those who use the output perform the process. Classic organization structure defines departments that provide goods and services for other departments. Changes in information technology can facilitate integration across departmental barriers so that those who use the output can perform the activities necessary to do the process. This principle can also greatly increase the support of those performing the process, which can set the stage for further process improvements.

- Subsume information processing work into the real work that produces the information. Rather than generating information from processes through "accountants," a user (worker) could generate information from the "real" work process. A good example of this is the development of information using scanners on bar codes, which allows the worker on the loading dock to capture information as part of the normal work routine. Organizational design of this type eliminates the scenario of different "accountants" preparing information for other accountants, cascading information through organization.

- Treat geographically dispersed resources as though they were centralized. Information technology allows firms to virtually centralize their resources (e.g., departments), aggregating the resources of multiple locations. Economies of scale are then used to decrease costs.

- Link parallel activities instead of integrating their results. Parallel processes, typically done by multiple persons, are combined in a single job, rather than trying to assemble multiple disjointed efforts after they are completed. In some situations, some of the activities can be eliminated. In others, assembling the results of the parallel activities becomes less of a task, because activities are assembled as they occur.

- Put the decision point where the work is performed. In many organizations and processes, those who do the work are different from those responsible for making decisions about the work. This principle indicates that people who do the work, possibly supported by IT, should be allocated decision-

making rights or responsibilities.

- Capture information once and at the source. Information that is gathered at the source has fewer chances of having error introduced into it. Rather than having "accountants" generate information from processes, which is then sent to other accountants, this principle focuses on generating the information at the time it is developed. Electronic data interchange (EDI) data developed by the customer in formulating the order information can be used directly by the vendor, eliminating the need for duplicating data. 
Many of these principles focus on eliminating cascading information gathering, preparation, and regathering and repreparation, through the use of IT. Using these principles in practice can guide a reengineering effort and make it significantly more productive, in turn making the business more productive and profitable. Not surprisingly, one result is that often fewer people are needed in a reengineered process. For example, when Ford Motors reengineered its procurement process it reportedly was able to reduce the number of accountants in the procurement process from 500 to 125 . Although corporate downsizing can be painful to those whose jobs are lost, typically it is positive from a process point of view.

\section{What Are the Roles of Tools and Artificial Intelligence in Reengineering?}

Reports of such large reductions in personnel and, accordingly, costs, have led a number of firms to pursue reengineering of business processes. To facilitate reengineering, a number of tools have been designed to help an analyst develop a model of workflow of the processes being investigated. Then, that model is analyzed to determine how the process can be reengineered.

What, then, is the role that we can expect artificial intelligence to play in business process reengineering? Hamscher (1994a) notes that there are two basic roles. First, most frequently expert systems and other artificially intelligent systems are used as enabling technologies to reengineer around outcomes. AI-based systems are used consistently with the principles of reengineering in order to improve the decision-making abilities of workers. This usage allows decision making to occur closer to where the work is performed or makes individuals' performance of multiple parallel activities easier, allowing those who use the output to perform the process. For example, insurance claims might be processed more efficiently and effectively by providing workers with an expert system that helps them perform many facets of their job.

Second, and the focus of this investigation, AI can be used as a tool in the reengineering process itself. AI can be built into the tools that are used to help reengineer processes. AI then is used to facilitate and support the reengineering design and can be used to capture reengineering knowledge for subsequent reuse.

\section{How Is Artificial Intelligence Used in Reengineering Tools?}

In order to assess the extent to which AI is used in tools designed to facilitate or support reengineering and to ferret out which applications involving business process reengineering tools use AI, tool developers were surveyed. Conducted during January through March 1996, the survey was sent by e-mail and fax to a number of developers of reengineering tools that were found on any three comprehensive lists of business process reengineering tools. 2 In order to ensure that the products were commercial, addresses for products listed at universities were not sent the survey.

The survey was primarily concerned with the use of AI and the success of its use in the business process reengineering tools. The survey was also aimed at determining the basic nature of knowledge representations, e.g., case-based or network-based. In addition, the survey also solicited respondents' comments to open-ended questions, such as the advantages and disadvantages of using AI in reengineering tools.

Some survey results are summarized in Table 1. Results indicate that few firms are marketing AI-based tools to support reengineering. 


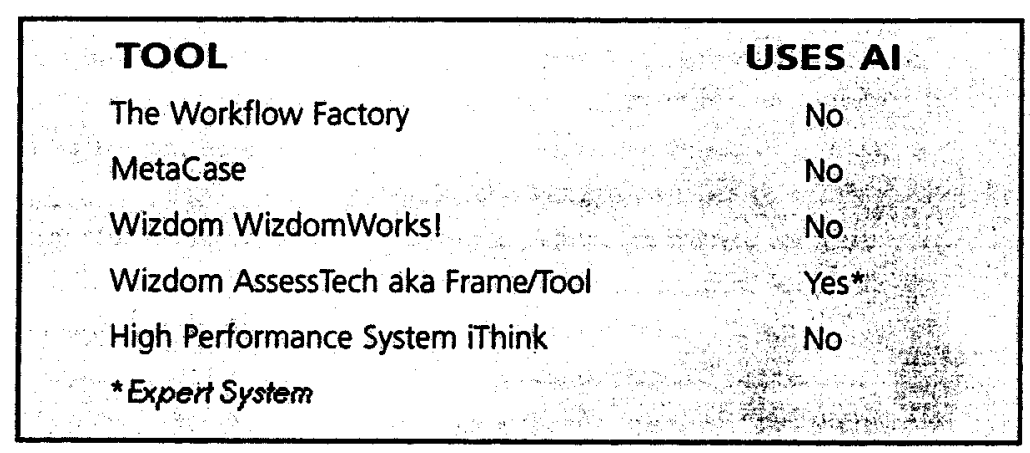

Table I. Al capabilities of reengineering tools

However, there are some exceptions. Wizdom Systems developed an expert system to help determine if firms are ready to adopt a new technology. The expert system AssessTech (Pierotti 1996), designed for use by hospitals, uses a knowledge-based approach to guide the user through analysis of a number of factors in order to measure the readiness of an organization to pursue the implementation of a technology and to identify optimal strategies for pursuing the implementation. Another version of the same system was developed for use by the Air Force.

Commercial tool developers have not disclosed the use of AI, if in fact the tools do contain AI, for many reasons. Issues such as competitive advantage may lead to nondisclosure of such information. Some developers may be more concerned with making sure that the tool does the job, not whether it contains AI. However, it may be that many BPR tools do not use AI. If that is the case, perhaps some of those tools could benefit from some of the AI-based approaches that have appeared in the literature.

\section{What Are Some Artificial Intelligence-Based Approaches to Reengineering Tools?}

Most of the research using AI and expert systems to model and support reengineering processes first appeared in a 1994 workshop on artificial intelligence in business process reengineering (Hamscher 1994a). The research discussed at that workshop and some subsequent research, virtually all deriving from practice, falls into five researcher-defined categories: (1) workflow analysis using network models of processes, (2) qualitative reasoning, (3) AI-based simulation, (4) knowledge-based systems, and (5) case-based reasoning.

1. Workflow analysis using network analysis or graphical analysis. An important approach to reengineering is to analyze current workflow processes and try to find improvements to those work flows. Typically workflow processes are mapped into a network model that an AI-based system then analyzes. Differences between systems occur with what view is (or views are) captured in the networks. Generally, systems based on network analysis are fairly generic, designed to reengineer work flows from a number of domains. Accordingly, these systems contain no domain- or process-specific knowledge.

Huang and Richter (1994) of Boeing Computer Services developed a network-based prototype system designed to simultaneously model flows and resources in business processes. Their system, CAPEESH, allows multiple levels of abstraction, ranging from executive-level overviews to detailed workflow analysis. CAPEESH allows multiple 
views of the models, including resource-based, time-based, and agent-role-based (e.g., skills). Since many processes appear to be similar or use similar subprocesses, CAPEESH also allows the reuse of models, captured as templates. In addition, Huang and Richter's system employs a user interface mechanism that allows the user to focus on only portions of any of the networks.

Jain et al. (1994) of Andersen Consulting built a prototype called Structured Process Diagram (SPD). SPD is an enhanced flow diagram showing activities in a process (tasks and decisions); work objects (that have states, flow between activities, or are inputs and outputs to activities); and agents (initiators, internal operations, and recipients). Given the topology offered by these three components, Jain et al. provide a number of heuristics that the system can use to redesign a process. These heuristics include changes to the activities, such as aggregation, reassignment, or modification of the concurrency of the activities.

Yu and Mylopoulos (1994 and 1996) of the University of Toronto presented a workflow analysis using network analysis, using an example modeled for medical insurance claims. Network models are used to plot information about generic goals, tasks, resources, and their relationship with actors. Three different models of the same process capture different views of the organizational environment. These models, and the AI embedded in them, are as follows:

- Workflow model, which describes what actors take what entities as inputs and what entities those actors produce as outputs.

- Actor or "strategic dependency" model, which captures resource and task dependencies as well as goal dependencies. In this model, actors can be represented as agents, each with goals and resource and task dependencies.

- Functional or "strategic rationale" model, which models the means-ends relationships in the particular process, using goals, tasks, resources, and flows. In this model, goals and rules are used to support explicit means-ends reasoning to determine the feasibility of goals. In particular, the network of means-ends relationships can be viewed as a "plan" for meeting specific goals. A plan, in this case, consists of resources, tasks, and subgoals, connected by arcs representing the means-ends links. Means-ends arcs are seen as instantiations of rules for achieving the functional goals.

2. Qualitative reasoning In addition to the three approaches discussed in Item 1, a fourth approach, formulated by Yu and Mylopoulos (based on work by Mylopoulos et al., 1992), is qualitative reasoning. Although the other three approaches discussed earlier allow users to determine the feasibility of accomplishing goals, the qualitative reasoning approach allows them to determine the contribution toward accomplishment of the goal, whether positive or negative.

Yu and Mylopoulos's qualitative model compared the impact of using an expert system and that of using a human medical adviser. The model focused on three decision variables resulting from the choice: cost, speed of service, and decision quality. Intermediate variables that directly influenced the decision variables include development cost, operating cost, depth of knowledge, and turnaround time. Qualitative reasoning then measures whether the contribution to the intermediate and decision variables was negative or positive, what variables where influenced, the impact of multiple contributors (using "and" or "or"), and the extent of the influence. 
3. AI-based simulation (agents and model-based reasoning). Although they may also have a qualitative component, simulation models are generally characterized by a graphical network representation of a system, coupled with a simulation mechanism that allows the models to change over time. Quantitative analysis of system dynamics is coupled with the more intuitive graphical presentation of change over time. Several authors have discussed the use of AI-based simulation models.

For example, Klein (1994) of the Mitre Corporation briefly discussed the use of simulation models, with changes in behavior and organizational relationships, to model qualitative states over time. In a brief description of work in progress, Klein described the use of interacting agents that facilitate evolution of the system over time in a model in which decision makers explore and adopt alternative behaviors.

Kant and Young (1994) of Schlumberger Austin Research briefly argued that simulation models must be hierarchical and that AI can be used to provide a framework for that hierarchy. They argued that " $[\mathrm{t}]$ he key role for $\mathrm{AI}$ is in representing the information about the different levels and knowledge about how to move between them." Model-based reasoning was seen as a way to model, explain, and reuse knowledge about processes.

Rock and Yu (1994) of Coopers \& Lybrand discussed a system called SPARKS (System Performance Analysis Using Real-Time Knowledge-based Simulation), which is based on Gensym's G2 expert system shell. SPARKS has three components: a process flow model; a resource model consisting of agents, machines and system resources; and work volumes and descriptions. Once a model is developed it can be used to simulate dynamic behavior, capturing a broad range of model-based metrics on productivity, cost, and quality. SPARKS also can be used to model the formal and informal knowledge, skills, and competencies that must be embedded in any reengineered system. Gensym's ReThink appears to have similar design and capabilities (see http:// www.gensym.com/).

4. Knowledge-based systems. Selfridge and Terveen (1996) of AT\&T Bell described a knowledge-based system known as the Software Design Assistant (DA). DA was developed with a "process owner" in a large software development organization to solve a particular problem: the vast amount of informal "folklore knowledge" of software development was overwhelming the process guidelines. This knowledge (for example, performance constraints, properties of current implementation, impact of design decisions on other aspects of the software, local programming conventions, and personnel and organization constraints) was captured in DA and used to drive a dialog with the software developer. The dialog would determine characteristics of the current software task and knowledge appropriate to that task. DA was fully integrated with the software process and included a novel "maintenance subprocess" to ensure that the knowledge remained current. DA was successfully deployed in the organization and succeeded at streamlining and improving a critical process.

5. Case-based reasoning. Ku et al. (1996) of Battelle developed an interactive, intelligent system for reengineering. Their system uses previously encountered situations, modeled as case-based reasoning to facilitate business reengineering. For a particular reengineering situation, the system retrieves the case from its case repository that best meets the needs of the current situation. It then refines the case to meet the specific needs of the current situation. A simulation model then is used to evaluate different solutions generated by the system.

Previous research on integrating AI and reengineering has employed a number of 
types of knowledge representations (e.g., rule-based and case-based) and AI approaches (e.g., AI and simulation). Generally, the systems built to date are aimed at reengineering generic processes; however, some systems have focused on particular industries or domains, such as hospitals or software design. As a result, the proposed approaches are all general, applicable to a wide range of processes. Systems ignore the prospect of including in their systems knowledge about specific processes (e.g., procurement systems). This lack of specificity has led to not exploiting an important advantage of knowledge-based systems and AI-the ability to incorporate domain knowledge. A system discussed in the next section fills some of that gap by using knowledge-based systems to exploit reengineering knowledge about a specific business process.

What Is a "Best Practices" System?

"Best practices" refers to the "best" way to perform some activity. Reengineering has led to permanent changes in our knowledge of how to perform some business processes more efficiently. As a result, as generic processes become reengineered, best practices begin to emerge. These best practices then are adapted by competitors so that they can remain competitive. In some cases the best practices are adapted by suppliers and customers so that their systems remain consistent with the firm that is employing the best practice.

Recently there has been some concern with the notion that a best practice exists. Unfortunately, the term seems to imply an absolutism about the relative quality of the practice compared with other versions of the process. As a result, there has been a movement (see, for example, http://wwwfar.npr.gov:80/BestP/FAQ_Practice.html) to refer to these best practices as "promising" practices.

We suggest that one way to capture, maintain, manage and further diffuse knowledge associated with these best practices is to use a knowledge-based system. In order to illustrate the ability of knowledge-based systems to capture best practices, in the next two sections we describe an application used at many firms around the country and a prototype knowledge-based system that captures process knowledge. The system was built to test the notion that best practices reengineering process knowledge could be captured as a knowledge-based system for analysis and reuse.

\section{What Is a Knowledge-Based System Approach to Reengineering?}

System Design. The focus of the particular system we discuss here is the goods ordering and payment process, sometimes referred to as the "procurement process" or "accounts payable process" (e.g., Hammer 1990). Procurement was chosen because it is relatively generic in many ways and because virtually all organizations employ a procurement process. In addition, the reengineering of procurement by researchers such as Hammer has led to a basic change in the "generally accepted" procurement process. Although the system was designed for reengineering a single process, the approach could be extended to other, related operational and financial processes for which there has been a similar advancement of the knowledge of how organizations best perform that process.

This system is unlike previous efforts to integrate AI and reengineering because it includes substantial application (procurement reengineering) knowledge that is based on the premise that the basic knowledge about how organizations should perform this process has changed. Further, the system was loosely built on the basis of first principles (Davis 1983), in particular, Hammer's "principles of reengineering." Like many other knowledge-based systems, this system is diagnosis-based (e.g., Buchanan and Shortliffe 
1983 ) in that it searches for "symptoms" of problems and then proposes "cures." In this case, the system is designed to look for signals that indicate that a process needs reengineering and then provides a recommendation based on the symptom it was given.

The system was designed in seven modules, each corresponding to the seven principles of reengineering. This subsection discusses the design of the systems, in terms of procurement knowledge for each of these principles of reengineering, and the next subsection summarizes some of the aspects of implementing the system and illustrates it with selected excerpts from the knowledge base.

1. Organize by outcomes, not tasks. Situations are analyzed that indicate that organization is based on tasks and not outcomes. Some signals of task organization are given through the number of agents involved with the transactions or activities associated with a process. Other signals of task organization are given through the number of agents that are responsible for a single vendor or agent. For example, in a procurement system, if more than one person is involved in purchasing from the same vendor, the focus may be too specific to purchasing tasks. Another signal is the basis on which those agents are evaluated; for example, are they evaluated by measures that lead directly or indirectly to the outcome? In a procurement system, task evaluations might include number of partial purchase orders generated or purchase orders corresponding to receiving documents. The system treats these signals as symptoms of potential areas of reengineering.

2. Have those who use the output perform the process. Matching work performed and information used is critical to assigning responsibility and generating quality output. For example, in the procurement application, the purchasing agent is one of the primary users of purchase order data. Accordingly, the purchasing agent performs the process and is responsible for the quality of the purchase order data. As a result, when the procurement system is reengineered the purchasing agent becomes responsible for getting the purchase information into the firm's database. The system tries to match the output and work as a basis of determining what needs to be reengineered in the existing procurement system.

3. Subsume information processing into the real work. Many "accountants" simply reprocess information developed by others into a form that is acceptable to the firm's financial and operational systems. Systems evolve over time with this reprocessing in mind by accommodating sequentially messaged information. Reengineering focuses on eliminating reprocessing of information, seeking to gather it from the work. Thus, as noted by Hammer (1990, p. 110), "Ford's redesigned accounts payable process embodies the new rule. With the new system, receiving, which produces the information about the goods received, processes this information instead of sending it to accounts payable."

Reengineering seeks to eliminate redundant sources of information. For example, invoices, which are generated by the vendor's accounting system, and purchasing agreements, which are generated by the purchasing department, contain basically the same information. We can eliminate one of the sources of information (invoices), focusing on the legal contract established in the purchasing agreement that is developed as part of the purchasing process. As a result, a reengineered procurement system typically includes only information gathered from purchasing (what is expected) and from the receiving dock (what was actually received).

The system asks questions that attempt to determine if information is gathered through the work or if there are accountants that reprocess this information. If such sit- 
uations are found, they form the basis of recommendations for reengineering.

4. Treat geographically dispersed resources as though they were centralized. Economies of scale can be generated by virtually centralizing resources. In terms of the procurement process, this module is primarily concerned with determining the possibility of centralizing the procurement process. As a result, the knowledge in this module focuses on finding ways to either actually or virtually centralize procurement activity.

5. Link parallel activities instead of integrating their results. Invoices and purchase orders are parallel sources of what should be the same information. If both are used, differences that provide a potential source of anomaly or error must be resolved. As a result, in the reengineered procurement process, only one of those processes is used, the legalbased purchase order information.

The system has knowledge of these parallel activities. For example, the system knows that if invoices are used the process can be reengineered to exclude the invoice. The system searches out documents, using its knowledge of the procurement process and related documents, with the goal of eliminating parallel development situations.

6 . Put the decision point where the work is performed. The reengineered procurement process places decision making at the source, supplementing decision makers with technology, such as expert systems. For example, before inventory is accepted at the receiving dock, as noted by Hammer (1990, p. 110), "[t] he new computer system can easily compare delivery with the order and trigger the appropriate action." Accordingly, the system contains knowledge about where different kinds of decisions should be made in a classically reengineered procurement system.

In addition, the comparison made by the computer eliminates the need for an accountant compare information about the receipt of goods with reports about ordering goods. The comparison is made by the computer at receipt. Because matching has been done at receipt, the reengineered system sends out a check when the goods are received, further reducing the need for accountants whose sole job is to reprocess information generated by others.

As a result, the system's knowledge is aimed at finding symptoms of where the decision point is far removed from where the work is performed. The system then provides recommendations on how to incorporate decision making into the work.

7. Capture information once and at the source. Information technology provides some significant capabilities to facilitate reengineering. Knowledge in this module is aimed at finding situations where technology is unused but could be used to subsume information processing into the work. Manual systems are amenable to potential redesign through a variety of technologies. Order information can be captured from electronic data interchange (EDI) orders. Information generated from the receipt of inventory at the warehouse docks can be gathered using scanners. Thus, the system generates data about whether information is gathered at its origin or whether it is messaged by accountants and cascaded through the organization.

System Development. The prototype system was built using M.4 (Cimflex Teknowledge, 1991) and employs a rule-based structure. The system was built to demonstrate that Hammer's (1990) principles of reengineering provide a basis for system development and to demonstrate that knowledge-based systems were an important medium for capturing the change in process knowledge brought about by reengineering. As currently developed the system has about 100 knowledge base entries, roughly corresponding to expert system style rules. The system does not employ the certainty factors promulgated in M.4, since most discussions of process reengineering are 


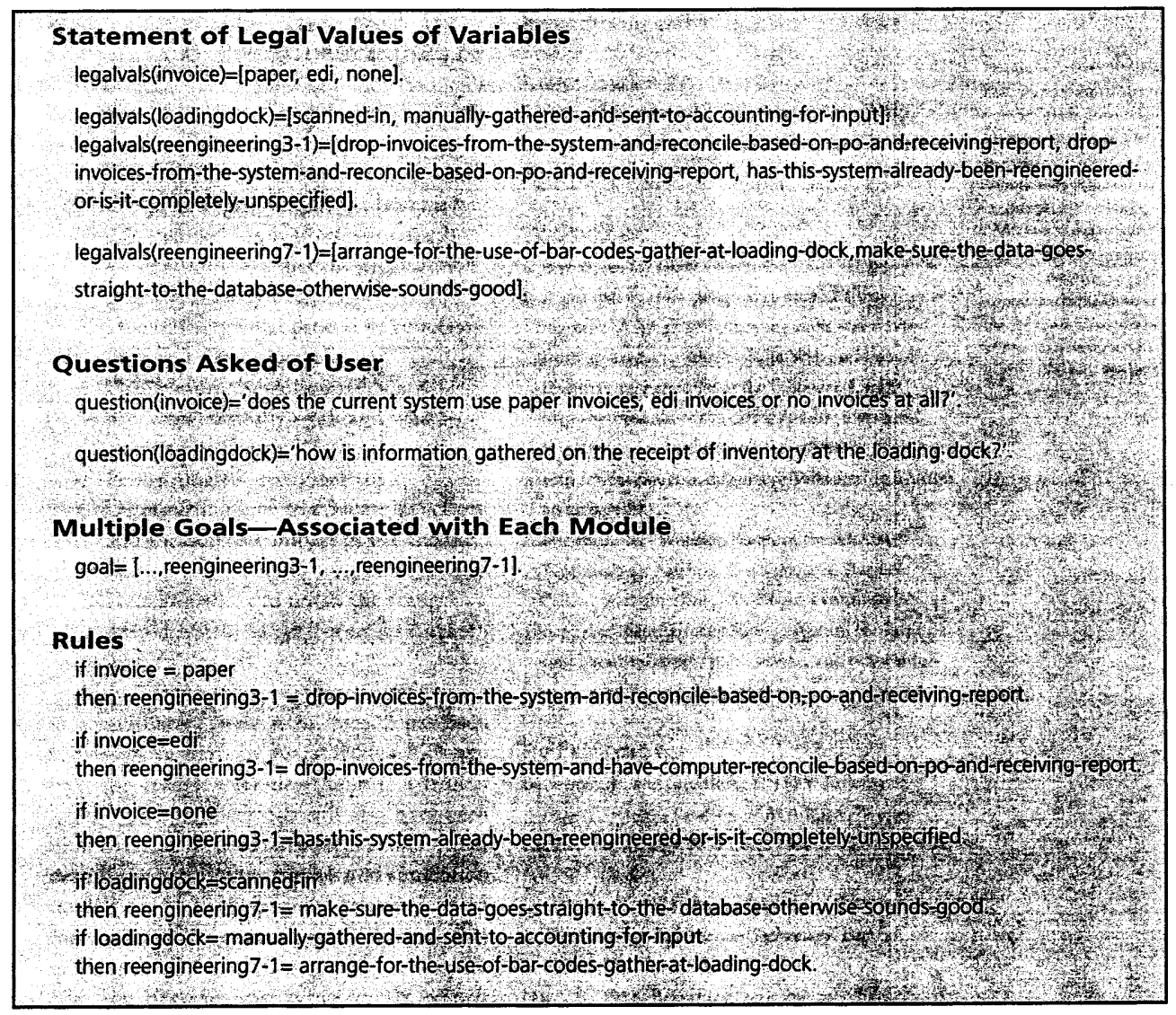

Figure I. Sample M.4 knowledge base for parts of two modules

couched in deterministic terms. Selected parts of the system's M.4 knowledge base are summarized in Exhibit 1.

Knowledge for the system was derived from the literature on procurement systems and reengineering, particularly Hammer's work on procurement processes, as well as from a human expert. As currently designed, the system takes into account reengineering that has been developed around what Hammer calls the "accounts payable system" or procurement. The system will not generate any novel solutions. Growth of the system's knowledge base requires the manual addition of new rules as well as other program content.

The system was validated through an enumeration of the possible paths and resulting consequences. No unusual behavior of the system was noted.

This system could be extended in a number of ways. Additional knowledge about related business processes, such as sales and collections processes, could be used to broaden the knowledge base. Industry-specific knowledge might be used to guide other changes. Alternative approaches to the rule-based approach also could be used. For example, case-based reasoning could be used to capture specific knowledge about particular reengineering projects of a firm's financial and accounting systems (e.g., Yu and Mylopoulos, 1996). Those cases could then be used to develop solutions to reengineer- 
ing problems by choosing the most similar case or by making some changes in the case to make it fit a new situation.

\section{What Steps Are Involved in the Al and Reengineering Life Cycle?}

One view of the basic reengineering life cycle involves the following seven steps:

1. Determining that reengineering is necessary in an organization (e.g., because foreign competition is affecting markets),

2. Determining whether the organization is ready to reengineer,

3. Determining what process or processes need to be reengineered,

4. Determining how the particular processes currently work and what needs to be changed,

5. Performing the reengineering,

6. Determining how to evaluate the effort and measure the impact of the change, and

7. Deciding when to revisit the reengineered system to see if it needs further work.

AI has been used to support reengineering in a number of these steps. Step 2 has been addressed, in part, by AssessTech, which can help the reengineerer determine if a firm is "ready" to reengineer. Systems also have been built that can be used with Steps 3 and 4 . For example, network flow models and simulation models can be used to model processes, assess the quality of the system, and determine what needs to be changed. Case-based systems can help the designer match previously reengineered systems to the current situations. Knowledge-based systems such as DA have been developed that provide folklore knowledge necessary to change a system. And knowledge-based systems (like the one developed in this paper) can be used to guide reengineering of generic processes where the basic underlying process knowledge has changed.

Step 5, although critical, is primarily a project management activity. Little information technology has been generated to support reengineering in Steps 1, 6, and 7, perhaps because of the inherent difficulty of the problems. However, work is in progress to develop technology-based tools to support these reengineering activities.

\section{Endnotes}

1. Boston Sunday Globe (November 12, 1995), p. A-127.

2. A number of tools designed to facilitate reengineering exist. A list of reengineering tools was derived from an extensive search of the Web and includes resources generated from the following URLs:

http://www.ie.utoronto.ca/EIL/tool/listbkp.html

http://www.ie.utoronto.ca/EIL/tool/other_BPR.html

http://www.phoenix.ca/bpr/bpr/Products/ (no longer an active link)

3. For example, "Working Notes of the AAAI-94 Workshop on Artificial Intelligence in Business Process Reengineering." See http://www.usc.edu/dept/ sba/atisp/AI/research/ai-reeng.htm for information about meetings, publications, and other information on $\mathrm{AI}$ and reengineering.

\section{References}

1. Buchanan, B. and Shortliffe, E. 1985. Rule-based Expert Systems, Reading, MA: AddisonWesley. 
2. Cimflex Technology, M.4 User's Guide, Palo Alto, CA, 1991.

3. Davenport, T. 1993. Process Innovation: Reengineering Work through Information Technology. Boston: Harvard Business School Press.

4. Davis, R. 1983. Reasoning from first principles. International Journal of Man-Machine Studies 19, pp. $9-16$.

5. Hammer, M. 1990. Reengineering work: Don't automate, obliterate. Harvard Business Review (July-August), pp. 104-112.

6. Hamscher, W. (ed.). 1994a. Working Notes of the AAAI Workshop on Artificial Intelligence in Business Process Reengineering. August.

7. Hamscher, W. 1994b. AI in business process reengineering: a report of the 1994 AAAI Workshop. AI Magazine 15, 4 (Winter), pp. 71-72.

8. Huang, M. and Richter, M.. 1994. Real World Process Modeling with G2. In Hamscher 1994a.

9. Jain, P., Liu, J. and Wagner, S. 1994. Transformational Approach to Business Process Redesign. In Hamscher $1994 \mathrm{~b}$.

10. Kant, E. and Young, R. 1994. Integrating Multilevel Models. In Hamscher 1994a, pp. 16-20.

11. Klein, G. 1994. Computer Simulation of Creative Adaptation to Business Process Reengineering. In Hamscher 1994a, p. 15.

12. Ku, S., Suh, Y. and Tecuci, G. 1996. Building an intelligent business process reengineering system: a case-based approach. International Journal of Intelligent Systems in Accounting, Finance and Management 5, 1.

13. Mylopoulos, J., Chung, L. and Nixon, B. 1992. Representing and using nonfunctional requirements: a process oriented approach. IEEE Transactions on Software Engineering 18, 6.

14. Pierotti, S. 1996. AssessTech: Assessment and Expert System for Evaluating Human Issues in Implementing New Technologies for the Healthcare Industry. Wizdom Medical Systems (March 15).

15. Rock, D. and Yu, D. 1994. Improving business process redesign. AI Expert (October), pp. $27-34$.

16. Selfridge, P. and Terveen, L. 1996. Knowledge management tools for business process support and reengineering. International Journal of Intelligent Systems in Accounting, Finance and Management 5, 1 .

17. Yu, E. and Mylopoulos, J. 1994. Organizational Modeling for Business Process Remodeling. In Hamscher 1994a, pp. 9-14.

18. Yu, E. and Mylopoulos, J. 1996. Using goals, rules and methods to support reasoning in business process reengineering. International Journal of Intelligent Systems in Accounting, Finance and Management 5, 1, pp. 1-13. 